\title{
Blocking of BDNF-TrkB signaling inhibits the promotion effect of neurological function recovery after treadmill training in rats with spinal cord injury
}

\author{
Xiangzhe $\mathrm{Li}^{1,2,3} \cdot$ Qinfeng $\mathrm{Wu}^{2,3} \cdot$ Caizhong $\mathrm{Xie}^{4} \cdot$ Can Wang ${ }^{5} \cdot$ Qinghua Wang ${ }^{6} \cdot$ Chuanming Dong $^{7} \cdot$ Lu Fang $^{1} \cdot$ \\ Jie Ding ${ }^{1} \cdot$ Tong Wang $^{1}$
}

Received: 5 January 2018 / Revised: 18 June 2018 / Accepted: 18 June 2018 / Published online: 12 July 2018

(c) International Spinal Cord Society 2018

\begin{abstract}
Study design Experimental study.

Objectives To investigate the role of BDNF-TrkB signaling that promotes the recovery of neurological function in rats with incomplete spinal cord injury (SCI) after treadmill training (TT).

Setting Rehabilitation Medicine Center of the First Affiliated Hospital of Nanjing Medical University, Nanjing, China. Methods Forty rats were divided into five groups: (i) Sham; (ii) SCI and phosphate-buffered saline (PBS) (SCI/PBS); (iii) SCI-TT/PBS; (iv) SCI/TrkB-IgG; and (v) SCI-TT/TrkB-IgG. The intrathecal catheter and T10 contusion SCI model was established. At 7-day post SCI, the BDNF-TrkB signaling was blocked by TrkB-IgG. Exercise began at 8th day after SCI and continued for 4 weeks. The BBB scale and motor-evoked potential (MEP) were used for the evaluation of the locomotor functions. The BDNF/TrkB, PSD-95, SYP synthesis, and neuroprotective effect was determined by western blot, Nissl, or immunohistochemistry staining.

Results The expression of BDNF and TrkB in the SCI-TT/PBS group was $1.46 \pm 0.09$ and $1.70 \pm 0.22$, respectively, higher than that in SCI/PBS group $(0.51 \pm 0.04$ and $0.76 \pm 0.07$, respectively), relative to the Sham group. The BBB scores in the Sham, SCI/ PBS, SCI-TT/PBS, SCI/TrkB-IgG, and SCI-TT/TrkB-IgG groups were 21.00 $\pm 0.00,7.63 \pm 0.74,12.13 \pm 1.36,7.88 \pm 0.64$, and $8.75 \pm 0.88$, respectively. The percentages of MEP responders/non-responders were $100,0,75,0$, and $50 \%$. The MEP latencies in Sham, SCI-TT/PBS, and SCI-TT/TrkB-IgG groups were $6.65 \pm 0.19,13.32 \pm 2.95$, and $19.55 \pm 4.55 \mathrm{~ms}$, respectively. The number of $\mathrm{NeuN}^{+}$neurons, the cell body area of motor neurons, PSD-95, and SYP expression in the SCI-TT/PBS group was significantly higher than that in the SCI/PBS, SCI/TrkB-IgG, and SCI-TT/TrkB-IgG groups.

Conclusion The BDNF-TrkB signaling is a critical pathway in exercise training that promotes the recovery of neurological function in rats with incomplete SCI.
\end{abstract}

Electronic supplementary material The online version of this article (https://doi.org/10.1038/s41393-018-0173-0) contains supplementary material, which is available to authorized users.

$\triangle$ Tong Wang

wangtong60621@163.com

1 Rehabilitation Medicine Center, The First Affiliated Hospital of Nanjing Medical University, 210029 Nanjing, China

2 Rehabilitation Medicine Center, Suzhou Science \& Technology Town Hospital, 215153 Suzhou, Jiangsu, China

3 Rehabilitation Medicine Center, Suzhou Hospital Affiliated to Nanjing Medical University, 215153 Suzhou, Jiangsu, China

\section{Introduction}

Spinal cord injury (SCI) is a traumatic neurological condition that causes abnormalities in locomotor, sensory, and autonomic nervous functions below the level of injury.

4 Department of Rehabilitation Medicine, Nanjing General Hospital, 210000 Nanjing, China

5 Children's Development Center, Suzhou Municipal Hospital, 215008 Suzhou, Jiangsu, China

6 Experimental Animal Center, Nantong University, 226021 Nantong, Jiangsu, China

7 Department of Human Anatomy, Medical College of Nantong University, 226021 Nantong, Jiangsu, China 
Approximately, 25-50 million individuals suffer from SCI each year worldwide, primarily due to car accidents, crashes, and violence, which severely affect the quality of life in patients and are a burden to the family and society [1]. Therefore, the therapies that promote SCI rehabilitation are under intensive focus.

After SCI, a marked spinal cord neuronal loss, axonal damage, inflammatory cell infiltration, astrocyte and microglia proliferation, nerve fiber demyelination, and a series of secondary changes may occur $[2,3]$. In the central nervous system (CNS), the self-repair mechanism is limited post injury, which restricts the effective reorganization and regeneration of the neurons and neural circuits, resulting in a limited spontaneous recovery of the function [4]. Owing to the lack of beneficial factors and effective stimulation in CNS that promotes neurogenesis and axonal growth after injury, the limited axonal growth and inability to form an effective neural circuit limits the recovery of the function [5]. Therefore, increasing the beneficial factors of nerve regeneration and internal environment for functional recovery after SCI is imperative.

Exercise therapy (such as treadmill training, TT) is a simple and easy method in SCI rehabilitation that promotes the overall functional recovery in animals and patients with SCI via reduced syringomyelia area and formation of glial scar, promoting axonal and synaptic regeneration, and neural circuit reconstruction [6-10]. The above effects may be related to the increased expression of neurotrophic factors (NTs), such as brain-derived neurotrophic factor (BDNF) after exercise training in animals or individuals with SCI [11, 12]. BDNF is involved in repairing the damaged spinal cord via activation of the specific receptor tropomyosin-related kinase B (TrkB) [13, 14]. Our previous study found that exercise training promotes the expression of BDNF and TrkB in the distal spinal cord and the recovery of locomotor function in rats with SCI [15]. However, whether the exercise-induced increased level of BDNF is essential in SCI functional recovery and neuroplasticity is yet unclear. In this study, we examined the role of BDNF induced by TT in rats with SCI, by blocking the BDNF-TrkB signaling.

\section{Methods}

\section{Experimental animals and grouping}

Forty adult female Sprague-Dawley rats (8-10-week-old, 230-250 g weight) were provided by Shanghai Sippr-BK Laboratory Animal Co. Ltd. All the rats were housed at a constant temperature of $22 \pm 2{ }^{\circ} \mathrm{C}$, humidity $50-60 \%$, free diet, and 12-h light/dark cycle. The animal experiments were performed in accordance with the Nantong
University Ethics Committee and the China Laboratory Animal Guidelines.

The rats were randomly divided into five groups: Sham $(n=8)$; SCI/phosphate-buffered saline (PBS) (SCI/ PBS, $n=8)$; SCI-TT/PBS $(n=8)$; SCI/TrkB-IgG $(n=8)$, and SCI-TT/TrkB-IgG $(n=8)$ before intrathecal catheter operation.

\section{Intrathecal catheter and spinal cord injury}

The intrathecal catheter operation was performed at 1 week before SCI [16], in order to observe whether it caused neurological damage by watching the behavior changes in rats. Briefly, all the rats were administered an analgesic (acetaminophen, $50 \mathrm{mg} / \mathrm{kg}$, i.m.) $15 \mathrm{~min}$ prior to surgery [17], followed by anesthesia with $10 \%$ chloral hydrate $(0.3 \mathrm{~mL} / 100 \mathrm{~g}$ body weight $)$ by intraperitoneal injection. A 6-cm polyethylene PE-10 catheter [inner diameter (ID): $0.28 \mathrm{~mm}$; outer diameter (OD): $0.61 \mathrm{~mm}$, Smiths Medical International Ltd., UK] was sterilized, filled with PBS, and then, inserted about $2 \mathrm{~cm}$ between the third and fourth lumbar vertebra; therefore, the end of the catheter corresponds to the lumbosacral enlargement of the spinal cord. After the intrathecal catheter, $20 \mu \mathrm{L}$ PBS was used to irrigate the catheter using a $25 \mu \mathrm{L}$ microinjector (Model 1702, Hamilton company, GR, Switzerland), and the catheter was closed immediately. The rats were housed one per cage after intrathecal catheter. Penicillin $\left(2 \times 10^{6}\right.$ IU/rat, i.m. $)$ was used to prevent infection in skin and soft tissue wounds. The antibiotic and analgesic, mentioned above, were administered 1 time/day for 3 consecutive days. On the 7 th day after operation, $20 \mu \mathrm{L}$ of $2 \%$ lidocaine hydrochloride and PBS were injected along the catheter using a $25 \mu \mathrm{L}$ microsyringe, and the catheter was closed immediately. Subsequently, all the rats were paralyzed in both hindlimbs and tail, which could confirm that the catheter was unobstructed and appropriately placed.

After 7 days of intrathecal catheter, all rats were administered analgesic and anesthesia as above. The New York University (NYU) Impactor System is the modified Allen's method that was employed to establish a T10 incomplete SCI model by dropping a device rod $(10 \mathrm{~g})$ from a vertical distance of $2.5 \mathrm{~cm}$ onto the exposed spinal cord [15, 18]. The Sham group only exposed the T10 spinal cord. All the animals were injected one time intraperitoneally with normal saline $(2 \mathrm{~mL})$. Trimethoprim $(0.85 \mathrm{mg} / \mathrm{kg}$, i.m.) was used to prevent urethral infection. The antibiotic and analgesic, mentioned above, were administered 1 time/day for 3 consecutive days. After SCI, the bladder was voided by gently massaging the lower abdomen two times daily, until reflexive or voluntary emptying occurred. 
Intrathecal administration of TrkB-IgG

After 7 days post SCI, all the rats were administered analgesia and anesthesia as described above. The Alzet Osmotic pumps (model 2002, Alzet, Cupertino, CA, USA) were incubated with saline for $12 \mathrm{~h}$ at $37^{\circ} \mathrm{C}$ before insertion. Recombinant human TrkB-Fc chimera (TrkBIgG, R\&D Systems, Minneapolis, MN, USA) was used to block the BDNF-TrkB signaling. PBS alone or TrkB-IgG solubilized in PBS $(0.25 \mu \mathrm{g} / \mu \mathrm{L})$ was filled into the pumps [19, 20]. A 1-cm PE-50 (ID: $0.58 \mathrm{~mm}$; OD: $0.96 \mathrm{~mm}$; Smiths Medical International Ltd.) had attached the pumps, and the PE-10 catheter was inserted before. Preceding attachment, $20 \mu \mathrm{L}$ PBS was injected via the catheter using a $25 \mu \mathrm{L}$ microinjector to observe the catheter patency. Finally, the pump and polyethylene catheter were placed in tissue pocket subcutaneously on the back of the rats, and the solutions could reach the subarachnoid space of the lumbar spinal cord about $8 \mathrm{~h}$ later. The TrkB-IgG was pumped continually at a rate of $3 \mu \mathrm{g} / \mathrm{day}$ for 4 weeks. Since the in vivo stability of TrkB-IgG was observed 2 weeks [21, 22], an identical procedure was performed to replace the pumps after 3 weeks in all the groups. Penicillin and analgesic were administered as described above.

\section{Treadmill training}

The TT began at 8 days post SCI and was conducted for 5 days/week and 2 times/day for 4 consecutive weeks [15]. The exercise training was performed for $20 \mathrm{~min}$ each time based on the body weight supported TT. The support range was set as $20-40 \%$ of rats' weight, and we reduced the support gradually along with the recovery of hindlimb function (i.e., when BBB score was $\leq 8$, the support was $40 \%$ of its weight; when BBB score was 9, the support was $35 \%$ of its weight; when $\mathrm{BBB}$ score was 10 , the support was $30 \%$ of its weight; and when BBB score was $\geq 11$, the support was $20 \%$ of its weight). The speed of the treadmill was set to $6 \mathrm{~m} / \mathrm{min}$ [3]. The bladders of all the rats were drained before each training session.

\section{Evaluation of the hindlimb locomotor function}

To evaluate the motor function of the hindlimbs, the Basso, Beattie, and Bresnahan (BBB) scale [23, 24] were used in an open field $\left(80 \times 130 \times 30 \mathrm{~cm}^{3}\right)$ preoperatively and at $1,3,7,14,21,28$, and 35 days post SCI [19]. All the rats were observed individually for $5 \mathrm{~min}$ by two observers, who were blinded to the allocation of the animals.
Evaluation of the motor-evoked potential (MEP)

To evaluate the function of axonal conduction of the corticospinal tract, we used the transcranial magnetic MEP (tcMMEP) at the end of the experiment (5th week post SCI) [25]. All the animals were anesthetized as above, and the magnetic transducer $(110 \mathrm{~mm}$ diameter, magnetic field strength $0.1-1.0 \mathrm{~T}$ ) was placed over the skull (Cz. point over the skull) and a short single magnetic pulse $(20 \mathrm{~ms}, 60 \%$ of the maximum stimulation intensity) was delivered by the magnetic stimulator (MagProX100 stimulator, Dantec medical, Denmark) attached to the handheld magnetic transducer (C-100, Dantec medical, Denmark). The electromyograph (EMG) responses were recorded by the EMG device (Keypoint portable 33A07, Dantec medical) from the monopolar needle electrodes inserted into the gastrocnemius. In this study, the amplitude measurements $>0.13 \mathrm{mV}$ and latencies $<25 \mathrm{~ms}$ were considered as physiological responses [25].

\section{Tissue preparation}

At the end of the experiment, four rats in each group were anesthetized by the inhalation of isoflurane and perfused intracardially with $200 \mathrm{~mL}$ normal saline, followed by $250 \mathrm{~mL}$ of $4 \%$ paraformaldehyde in $0.1 \mathrm{M}$ phosphate buffer (pH 7.4). Then, the lumbar segments of the spinal cord (L2-L5) [19] were fixed with 4\% paraformaldehyde for 12 h. Subsequently, the spinal cords were paraffin-embedded and sliced transversely into $5 \mu \mathrm{m}$ sections [26].

\section{Nissl staining}

The Nissl staining was used to observe the cell body area of motor neurons and morphological characteristics after SCI [15]. The L2-L5 spinal cord sections of all rats were deparaffinized and rehydrated, placed in cresyl violet for 30 min, and then, washed by running water, $95 \%$ alcohol for $2 \mathrm{~s}$, dehydrated, turned transparent, and mounted in neutral gums. The images of lamina IX in all spinal cord sections were captured by a digital microscope (Leica Microsystems, Wetzlar, Germany) and the cell body area of the gray matter motor neurons was analyzed using Image Pro Plus (Media Cybernetics Inc., Rockville, MD, USA).

\section{Immunohistochemistry staining}

After deparaffinization, rehydration, inactivation of peroxidase, antigen retrieval, and blocking with 5\% albumin from goat serum, the sections were probed overnight at $4{ }^{\circ} \mathrm{C}$ with the following primary antibodies: rabbit anti-neuron-specific nuclear protein (NeuN) monoclonal antibody (1:1000; Millipore, Darmstadt, Germany), rabbit anti-postsynaptic density protein-95 (PSD-95) polyclonal antibody (1:800; 
Abcam, Cambridge, MA, UK), and rabbit antisynaptophysin (SYP) monoclonal antibody (1:1000; Millipore). Then, each sample was washed with PBS and stained using secondary antibodies for $1 \mathrm{~h}$ at $37^{\circ} \mathrm{C}$. Finally, 3,3'diaminobenzidine- $4 \mathrm{HCl} / \mathrm{H}_{2} \mathrm{O}_{2}$ (DAB, Vector Laboratories, Burlingame, CA, USA) was used for visualization. Subsequently, the images of lamina IX in all the spinal cord sections were captured and analyzed by digital microscope and analyzed by Image Pro Plus.

\section{Western blot analysis}

The spinal cords of four rats in each group were removed and transversely cut at the lumbar enlargement (L2-L5) [19], followed by homogenization and centrifugation. The supernatants were proportionally diluted with $3 \times$ loading buffer and heated for $15 \mathrm{~min}$ at $37^{\circ} \mathrm{C}$. The samples were loaded onto $12 \%$ Tris/tricine SDS gels, transferred to PVDF membranes, and blocked with 5\% milk solubilized in TBST (pH 7.4, $10 \mathrm{mM}$ Tris- $\mathrm{HCl}, 150 \mathrm{mM} \mathrm{NaCl}$, and $0.1 \%$ Tween 20) at room temperature for $2 \mathrm{~h}$. Subsequently, the membranes were incubated with rabbit anti-BDNF monoclonal antibody (1:1000; Abcam), rabbit anti-TrkB polyclonal antibody (1:1000; Abcam), rabbit anti-PSD-95 monoclonal antibody $(1: 1000 ;$ Abcam), rabbit anti-SYP monoclonal antibody (1:1000; Millipore), or GAPDH (1:1000; Bioworld, Louis Park, MN, USA) at $4{ }^{\circ} \mathrm{C}$ overnight, followed by incubation with horseradish peroxidaseconjugated goat anti-rabbit IgG (1:2000, Vector Laboratories, Burlingame, CA, USA) for $1 \mathrm{~h}$ at room temperature. The immunoreactive bands were detected using the ECL plus detection system. GAPDH served as a loading control.

\section{Statistical analysis}

BBB scores were analyzed by repeated-measures analysis of variance and Bonferroni post hoc test. The quantitative data were analyzed by one-way ANOVA and post hoc Tukey's tests. $P<0.05$ was considered to be statistically significant. The number of MET responders/non-responders was analyzed by Fisher's exact test, and the statistical significance of the test was corrected to $P<0.005$ based on multiple comparisons. The above data were analyzed by SPSS 20.0 software (IBM, SPSS Inc., USA). The effect size and power analysis of BBB scores were conducted using $\mathrm{G}^{*}$ Power (version 3.1.9.2.) [27, 28].

\section{Results}

\section{BDNF/TrkB expression}

The expression of BDNF and TrkB in the SCI/PBS group $(0.51 \pm 0.04$ and $0.76 \pm 0.07$ relative to the Sham group, respectively) was lower than that in the Sham and SCI-TT/ PBS groups $(P<0.05)$. The expression of BDNF and TrkB in the SCI-TT/PBS group $(1.46 \pm 0.09$ and $1.70 \pm 0.22$ relative to the Sham group, respectively) was higher than that in the Sham and SCI/PBS groups $(P<0.05$, Fig. 1a-c).

\section{Locomotor function assessment}

Repeated-measures analysis of variance revealed a statistically difference between different time points $(P<0.001$, partial $\left.\eta^{2}=0.991\right)$ and the different treatment groups $(P<$ 0.001 , partial $\left.\eta^{2}=0.997\right)$. Moreover, a significant timegroup interaction was noted $\left(P<0.001\right.$, partial $\left.\eta^{2}=0.968\right)$. The SCI-TT/PBS group exhibited markedly enhanced locomotor recovery that began from the 2 nd week and continued up to the 5 th week as compared to the SCI/PBS, SCI/TrkB-IgG, and SCI-TT/TrkB-IgG groups $(P<0.05)$. In addition, the BBB scores of the SCI-TT/TrkB-IgG group were higher than those in the SCI/PBS group at the 5th week and SCI/TrkB-IgG group at the 4th and 5th weeks $(P<0.05)$ (Table 1).

\section{MEP assessment}

The percentages of MEP responders/non-responders in the Sham, SCI/PBS, SCI-TT/PBS, SCI/TrkB-IgG, and SCI-TT/ TrkB-IgG groups were $100 \%, 0 \%, 75 \%, 0 \%$, and $50 \%$, respectively. According to Fisher's exact test, no significant difference was noted in the MEP responders/non-responders between the Sham, SCI-TT/PBS, and SCI-TT/TrkB-IgG groups $(P>0.005)$; however, the MEP in these three groups was higher than that in the SCI/PBS and SCI/TrkB-IgG groups $(P<0.005$, Fig. 1d, e). The amplitude and latency of MEP wave in the SCI-TT/PBS and SCI-TT/TrkB-IgG groups were less than those in the Sham group $(P<0.05)$. Additionally, the MEP latency in the SCI-TT/PBS group was significantly shorter than that in the SCI-TT/TrkB-IgG group $(P<0.05)$, while no difference was detected in the amplitude between the two groups $(P>0.05$, Table 2$)$.

\section{Nissl staining}

In the Sham group, motor neurons in the ventral horn of the spinal cord exhibited an intact morphology, clear Nissl bodies in the cytoplasm, and abundant neuritis, while the motor neurons showed varying degrees of atrophy and Nissl body lysis in the other groups (Fig. 2a). The cell body area of the motor neurons in the Sham group was significantly larger than that in the other four groups $(0.61 \pm 0.08,0.86 \pm$ $0.06,0.59 \pm 0.12$, and $0.67 \pm 0.13$ in SCI/PBS, SCI-TT/ PBS, SCI/TrkB-IgG, and SCI-TT/TrkB-IgG groups relative to the Sham group, respectively, $P<0.05)$; the same in the SCI-TT/PBS group was significantly higher as compared to 

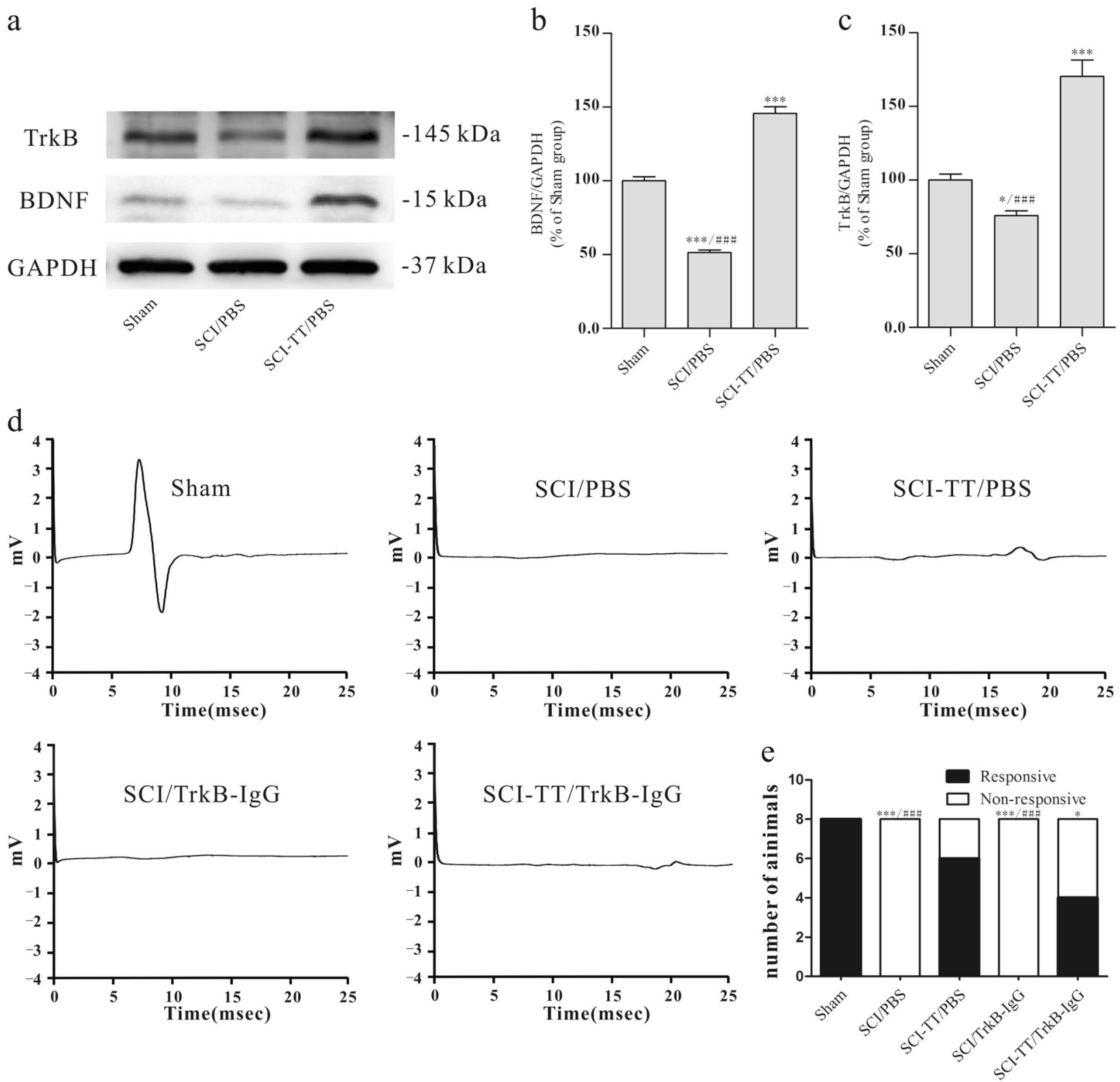

Fig. 1 Western blotting analysis of BDNF/TrkB and typical waveforms of transcranial magnetic motor-evoked potential in all groups. a The western blotting representative image of BDNF and TrkB in lumbar spinal cord. b The statistical graph of the relative density of BDNF. c The statistical graph of the relative density of TrkB. d Typical waveforms of transcranial magnetic motor-evoked potential response in all groups. e The number of responsive/non-responsive animals. Data presented as mean \pm s.e.m. Compared with Sham group, $* P<0.05$, $* * * P<0.001$; Compared with SCI-TT/PBS group, ${ }^{\# \# \# P<0.001}$

Table 1 BBB scores of all groups

\begin{tabular}{lllllllll}
\hline Group & Od & $1 \mathrm{~d}$ & $3 \mathrm{~d}$ & $7 \mathrm{~d}$ & $14 \mathrm{~d}$ & $21 \mathrm{~d}$ & $28 \mathrm{~d}$ & $35 \mathrm{~d}$ \\
\hline Sham & $21.00 \pm 0.00$ & $21.00 \pm 0.00$ & $21.00 \pm 0.00$ & $21.00 \pm 0.00$ & $21.00 \pm 0.00$ & $21.00 \pm 0.00$ & $21.00 \pm 0.00$ & $21.00 \pm 0.00$ \\
SCI/PBS & $21.00 \pm 0.00$ & $0.13 \pm 0.35$ & $0.88 \pm 0.35$ & $4.00 \pm 0.76$ & $5.88 \pm 0.83^{\mathrm{a}}$ & $6.75 \pm 0.89^{\mathrm{a}}$ & $7.25 \pm 0.46^{\mathrm{a}}$ & $7.63 \pm 0.74^{\mathrm{a}, \mathrm{b}}$ \\
SCI-TT/PBS & $21.00 \pm 0.00$ & $0.13 \pm 0.35$ & $0.75 \pm 0.46$ & $4.13 \pm 0.35$ & $7.88 \pm 0.99$ & $9.88 \pm 0.99$ & $11.00 \pm 1.41$ & $12.13 \pm 1.36$ \\
SCI/TrkB-IgG & $21.00 \pm 0.00$ & $0.13 \pm 0.35$ & $0.75 \pm 0.46$ & $4.00 \pm 0.53$ & $6.00 \pm 0.53^{\mathrm{a}}$ & $6.75 \pm 0.46^{\mathrm{a}}$ & $7.13 \pm 0.64^{\mathrm{a}, \mathrm{b}}$ & $7.88 \pm 0.64^{\mathrm{a}, \mathrm{b}}$ \\
SCI-TT/TrkB-IgG & $21.00 \pm 0.00$ & $0.25 \pm 0.46$ & $1.00 \pm 0.00$ & $3.50 \pm 0.53$ & $6.00 \pm 0.53^{\mathrm{a}}$ & $7.00 \pm 0.53^{\mathrm{a}}$ & $8.00 \pm 0.76^{\mathrm{a}}$ & $8.75 \pm 0.88^{\mathrm{a}}$ \\
\hline
\end{tabular}

Data presented as mean \pm s.e.m.

Compared with SCI-TT/PBS group, ${ }^{\text {a }} P<0.001$

Compared with SCI-TT/TrkB-IgG group, ${ }^{\mathrm{b}} P<0.05$ 
that in the SCI/PBS, SCI/TrkB-IgG, and SCI-TT/TrkB-IgG groups $(P<0.05)$. Furthermore, no significant differences were observed among the SCI-TT/TrkB-IgG, SCI/PBS, and SCI/TrkB-IgG groups (Fig. 2b).

\section{Immunohistochemistry staining of NeuN}

The number of $\mathrm{NeuN}^{+}$motor neurons in the Sham group was similar to that in the SCI-TT/PBS group $(20.75 \pm 4.00$ vs. $19.25 \pm 3.91$ ), without any significant differences $(P>0.05)$. Furthermore, the number of $\mathrm{NeuN}^{+}$cells in the

Table 2 Latency and amplitude of MEP waveforms

\begin{tabular}{llll}
\hline Parameter & $\begin{array}{l}\text { Sham } \\
(n=8)\end{array}$ & $\begin{array}{l}\text { SCI-TT/PBS } \\
(n=6)\end{array}$ & $\begin{array}{l}\text { SCI-TT/TrkB-IgG } \\
(n=4)\end{array}$ \\
\hline Latency $(\mathrm{ms})$ & $6.65 \pm 0.19$ & $13.32 \pm 2.95^{\mathrm{a}}$ & $19.55 \pm 4.55^{\mathrm{a}, \mathrm{b}}$ \\
Amplitude $(\mathrm{mV})$ & $5.35 \pm 2.64$ & $0.23 \pm 0.06^{\mathrm{a}}$ & $0.15 \pm 0.02^{\mathrm{a}}$ \\
\hline
\end{tabular}

Data presented as mean \pm s.e.m.

Compared with Sham group, ${ }^{\mathrm{a}} P<0.001$

Compared with SCI-TT/TrkB-IgG group, ${ }^{\mathrm{b}} P<0.01$
Sham and SCI-TT/PBS groups was significantly higher than that in the SCI/PBS, SCI/TrkB-IgG, and SCI-TT/TrkB-IgG groups $(13.50 \pm 3.61,12.83 \pm 2.72$, and $13.41 \pm 2.31$ in the SCI/PBS, SCI/TrkB-IgG, and SCI-TT/TrkB-IgG groups, respectively, $P<0.05$, Fig. 2a). However, no significant differences were detected among the SCI-TT/TrkB-IgG, SCI/PBS, and SCI/TrkB-IgG groups $(P>0.05$, Fig. $2 \mathrm{c})$.

\section{Immunohistochemistry staining of PSD-95 and SYP}

The relative density of PSD-95 and SYP was decreased significantly in all the SCI groups (PSD-95: $0.61 \pm 0.08$, $0.82 \pm 0.14,0.52 \pm 0.07$, and $0.62 \pm 0.12$; SYP: $0.61 \pm 0.13$, $0.85 \pm 0.08,0.48 \pm 0.11$, and $0.51 \pm 0.11$ in the SCI/PBS, SCI-TT/PBS, SCI/TrkB-IgG, and SCI-TT/TrkB-IgG groups relative to the Sham group, respectively, $P<0.05$, Fig. 3a). The expression was higher in the SCI-TT/PBS group than the SCI/PBS, SCI/TrkB-IgG, and SCI-TT/TrkB-IgG groups $(P<0.05)$. However, no significant differences were observed in the expression of PSD-95 and SYP among the SCI-TT/TrkB-IgG, SCI/PBS, and SCI/TrkB-IgG groups $(P>0.05$, Fig. 3c, d).
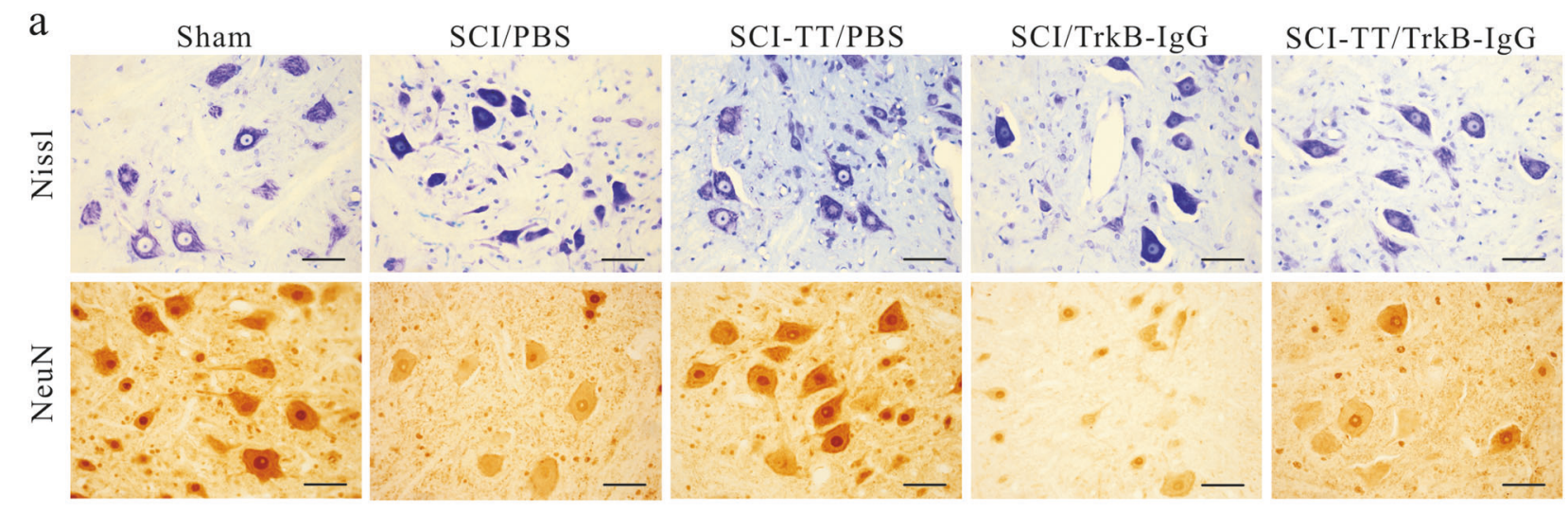

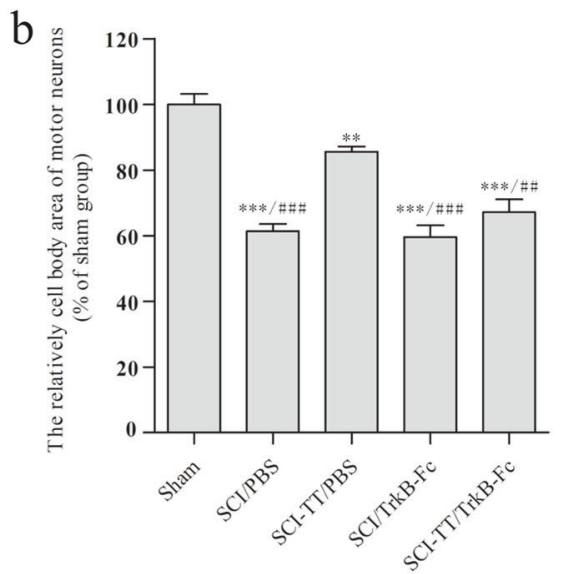

Fig. 2 Nissl staining and immunohistochemistry staining of NeuN. a The Nissl staining and immunohistochemistry staining of NeuN in lumbar spinal cord transverse section. b The statistical graph of the cell body area of motor neurons in the lumbar spinal cord.

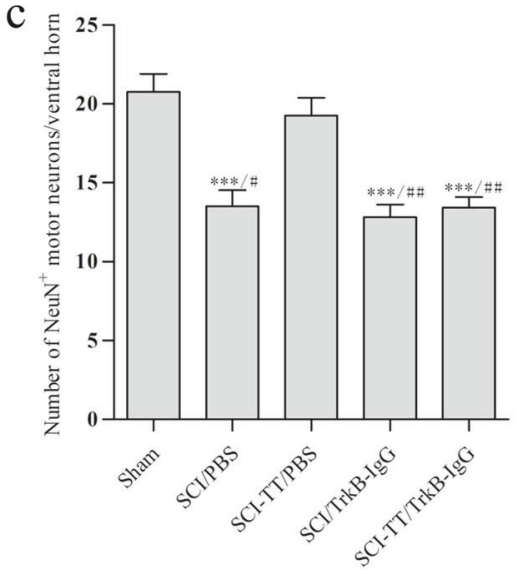

c The statistical graph of the number of $\mathrm{NeuN}^{+}$cells in the lumbar spinal cord. Data presented as mean \pm s.e.m. Compared with Sham group, $* * P<0.01$, $* * * P<0.001$; Compared with SCI-TT/PBS group, ${ }^{\#} P<0.05,{ }^{\# \#} P<0.01,{ }^{\# \# \#} P<0.001$. Scale bar: $50 \mu \mathrm{m}$ 


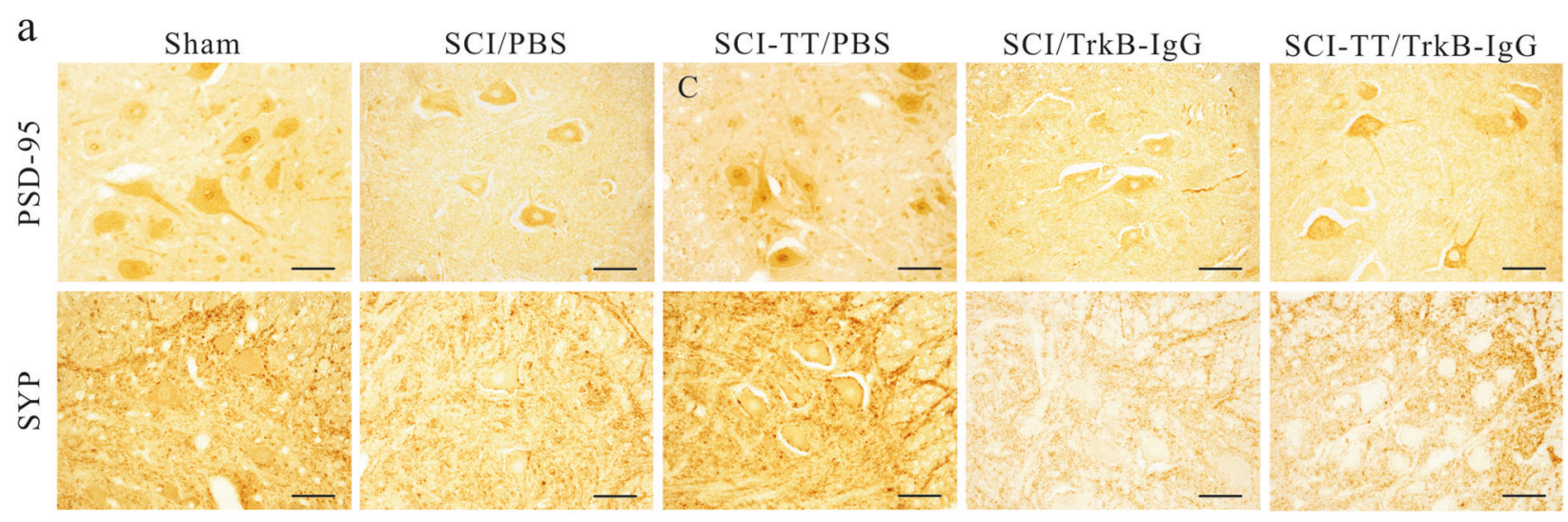

$\mathrm{b}$
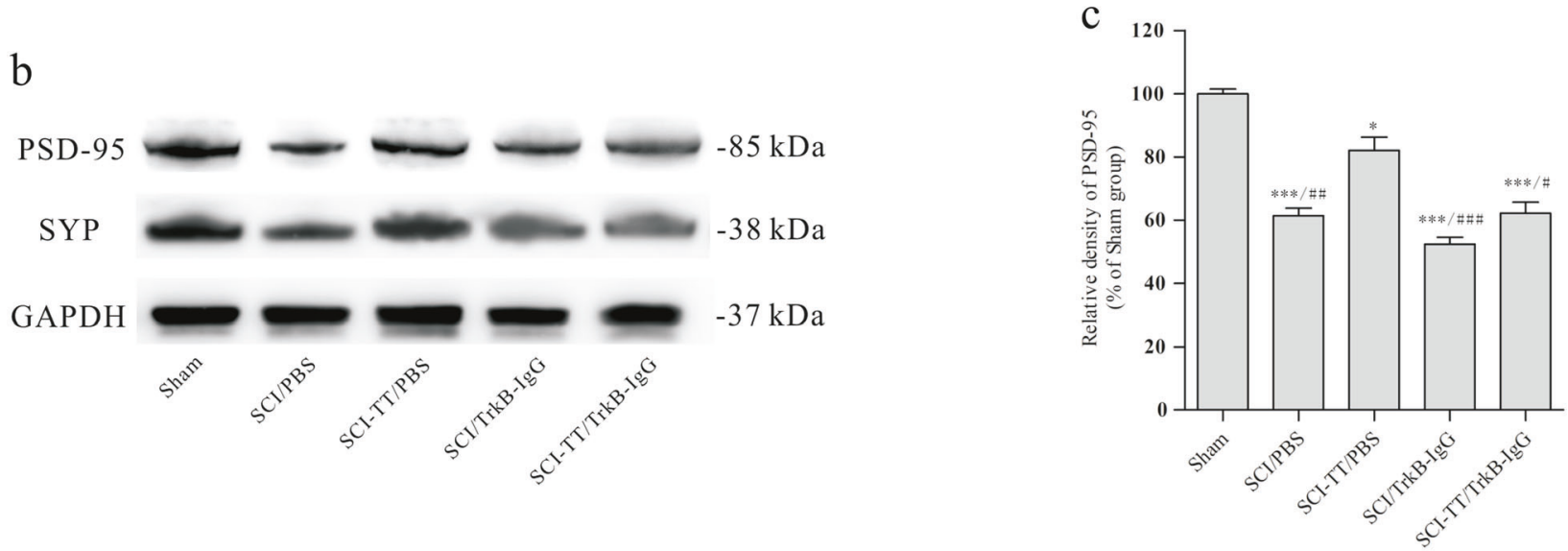

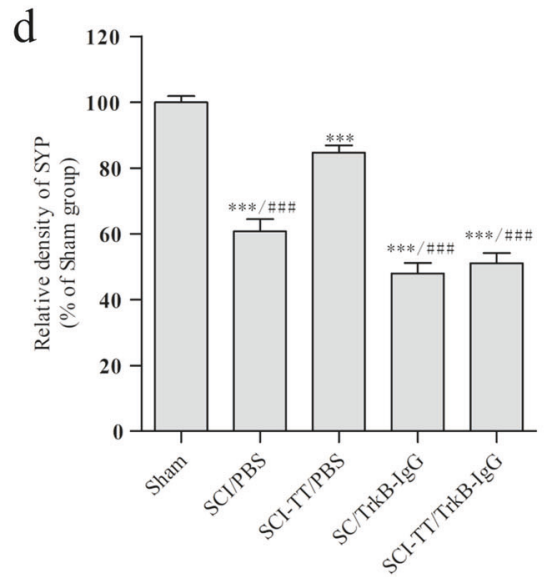

Fig. 3 Immunohistochemistry and western blotting analysis of PSD-95 and SYP. a The immunohistochemistry staining of PSD-95 and SYP in lumbar spinal cord transverse section. b The western blotting representative image of PSD-95 and SYP in lumbar spinal cord. $\mathbf{c}$ The statistical graph of the relative density of PSD-95. d The statistical

\section{Western blot analysis of PSD-95 and SYP}

The expression of PSD-95 and SYP proteins decreased significantly in all the SCI groups (PSD-95: $0.38 \pm 0.03$, $0.83 \pm 0.09,0.33 \pm 0.06$, and $0.40 \pm 0.05$; SYP: $0.43 \pm 0.08$, $0.76 \pm 0.12,0.27 \pm 0.05$, and $0.30 \pm 0.02$ in SCI/PBS, SCITT/PBS，SCI/TrkB-IgG， and SCI-TT/TrkB-IgG groups

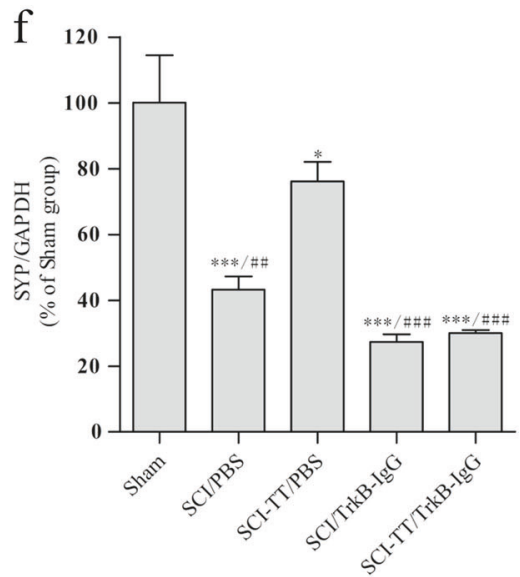

graph of the relative density of SYP. e Quantification of proteins PSD95. f Quantification of proteins SYP. Data presented as mean \pm s.e.m. Compared with Sham group, $* P<0.05, * * * P<0.001$; Compared with SCI-TT/PBS group, ${ }^{\#} P<0.05,{ }^{\# \#} P<0.01,{ }^{\# \# \#} P<0.001$. Scale bar: $50 \mu \mathrm{m}$

relative to the Sham group, respectively, $P<0.05$, Fig. $3 b$ ). Compared to the SCI/PBS, SCI/TrkB-IgG, and SCI-TT/ TrkB-IgG groups, the level of both proteins increased significantly in the SCI-TT/PBS group $(P<0.05)$. However, no significant differences were observed in either of the proteins among the SCI-TT/TrkB-IgG, SCI/PBS, and SCI/ TrkB-IgG groups (Fig. 3e, f). 


\section{Discussion}

As a noninvasive SCI rehabilitation therapy, exercise training increases the expression of BDNF $[6,8]$ and promotes the functional recovery of paralyzed muscles. Additionally, the remodeling of the brain, improvement of the spinal cord microenvironment, and protection of the function of remnant neurons via a variety of mechanisms is promoted [2]. However, to date, a majority of the studies have been focused on assessing the effect of exercise training on BDNF expression and locomotor function recovery, while the critical role in promoting the functional recovery through exercise training is ignored [11].

\section{Locomotor function}

The results showed that TT increased the expression of BDNF and TrkB and promoted the recovery of hindlimb motor function in rats with incomplete SCI. However, the promotion of locomotor function was inhibited significantly by blocking the BDNF-TrkB signaling. Tashiro et al. [19] observed that TT improved the motor function, spasticity, and pain; the blockage of BDNF-TrkB signaling by TrkBIgG inhibited its effects. The above results demonstrated that BDNF-TrkB signaling is a major pathway for exercise training-mediated promotion of locomotor recovery in rats with incomplete SCI. The overexpression of BDNF in the lumbar spinal neurons caudal to the lesion site after complete transection of the spinal cord increased the excitability of the lumbar spinal network, leading to robust and early locomotor recovery [29]. Thus, we speculated that combinational administration of BDNF coupled with exercise may be warranted as a potential therapy for the functional recovery of SCI in the future; however, further studies are essential for substantiation of these findings.

\section{MEP}

A previous study found that the latency of MEPs and severity of motor dysfunction showed a high correlation in patients with SCI [30]. In the present study, no effective MEP wave in the non-trained SCI rats was observed; however, the exercise training could promote the appearance of MEP waves, suggesting that it promotes the remodeling of the corticospinal tract and locomotor function recovery after SCI. Endo et al. [20] found that early exercise training promotes the regeneration of corticospinal tract neurons and the recovery of motor function in rats with SCI, which might be related to the BDNF-TrkB signaling pathway. Namsolleck et al. [31] found that the use of angiotensin-2 receptor agonists promote axonal plasticity, including corticospinal tract growth, by increasing the BDNF expression in SCI mice.
The recovery of motor function in untrained SCI rats might be related to the spontaneous recovery of central model generator (CPG) [15]. After exercise training, the conduction of corticospinal tract, $\mathrm{CPG}$ recovery, and synaptic plasticity might further improve the motor function. After blocking the BDNF-TrkB signaling, the number of MET responders in the SCI-TT/TrkB-IgG group was less than that in the SCI-TT/PBS group; however, no significant difference was observed between the two groups. This suggested that the BDNF-TrkB signaling may play a key role in exercise training promoting functional recovery by remodeling the corticospinal tract in rats with incomplete SCI, but it is not decisive.

\section{Neuroprotective effect}

In this study, Nissl staining showed that SCI can cause neuronal apoptosis, atrophy, loss of axons, and Nissl body lysis in the lumbar spinal cord. After exercise training, the cell body area of the motor neurons increased significantly, and the morphological features were similar to that of the Sham group as described in the previous studies [15]. Blocking the BDNF-TrkB signaling after exercise training significantly inhibited the above effect.

To further observe the function of motor neuron in the spinal cord, the number of NeuN-positive cells was determined. Lost/decreased production of NeuN might lead to the downregulation of alternative splicing of the RNA of target genes, thereby altering the complement of neuronalspecific gene expression [32]. Also, in this study, abundant NeuN-labeled motor neurons of the spinal cord were detected in the SCI-TT/PBS group. However, after blocking the BDNF-TrkB signaling, the number of NeuN-positive cells in the SCI-TT/TrkB-IgG group was significantly less than that in the SCI-TT/PBS group. This finding showed that TT is essential for maintaining the BDNF synthesis that can reduce the inactivity of the motor neuron injury and promote the neuroprotective effect after SCI.

\section{Synapse-related protein expression}

PSD-95 and SYP were labeled proteins constituting the postsynaptic membrane density protein and synaptophysin vesicle protein $[33,34]$ that marked the postsynaptic and presynaptic membranes, respectively. The results showed that the exercise training promoted the expression of PSD95 and SYP in the spinal cord of rats with SCI. When BDNF-TrkB pathway was blocked, the synthesis of PSD-95 and SYP in the SCI-TT/TrkB-IgG group was significantly reduced.

Ying et al. [21] found that after BDNF-TrkB signaling was blocked by TrkB-IgG, the effect of voluntary runner on restoring the motor function in rats with lateral cervical $(\mathrm{C} 4)$ 
hemisection was inhibited and the expression of synapsin I in spinal cord was decreased significantly as compared to the exercise group. This study indicated that after $\mathrm{C} 4$ hemisection, the voluntary runner promotes the motor function recovery and shapes the synaptic plasticity by increasing the BDNF levels. Long-term cultured hippocampal cells with BDNF also led to the increased synthesis of SYP, synaptobrevin, and synaptotagmin [35]. Yoshii et al. [36] found that PSD-95 is regulated by all the three pathways downstream of BDNF-TrkB signaling in the cortical neurons of mice. However, the mechanisms underlying the BDNF-TrkB signaling that promote synaptic plasticity are not yet elucidated.

\section{Conclusion}

Exercise training is an effective, noninvasive SCI rehabilitation therapy that promotes hindlimb motor function recovery, increases neuronal activity, and increases the lumbar spinal cord PSD-95 and SYP protein synthesis in rats with incomplete SCI; these effects are suppressed by blocking the BDNF-TrkB signaling. The current study showed that BDNF-TrkB is a critical signaling pathway in exercise training for the functional recovery, neuron protection, and enhanced synaptic plasticity in rats with incomplete SCI.

\section{Data archiving}

There were no data to deposit.

Acknowledgements We acknowledge Xin Chen and professor Jianzhong An for valuable advice. This work was supported by the National Natural Science Foundation of China (8117185, 81672258, and 81501189) and Livelihood Science and Technology program of Suzhou Science and Technology Bureau (SYS201785).

Author contributions XL was responsible for conceiving, designing, and performing the experiments, extracting and analyzing data, preparing the figures and/or tables, interpreting the results, and updating the reference lists. CX, CW, LF, and JD performed the experiments, screened the potentially eligible studies, and extracted data. CD and QW wrote and reviewed the drafts of the manuscript. TW and QW conceived the experimental design and reviewed the drafts of the manuscript.

\section{Compliance with ethical standards}

Conflict of interest The authors declare that they have no conflict of interest.

\section{References}

1. World Health Organization. International perspectives on spinal cordInjury[C]. Malta: (c) World Health Organization. 2013. http://www.who.int/mediacentre/factsheets/fs384/en/. Accessed 1 November 2017.

2. Fu J, Wang H, Deng L, Li J. Exercise training promotes functional recovery after spinal cord injury. Neural Plast. 2016;2016:1-7.

3. Jung S, Seo T, Kim D. Treadmill exercise facilitates recovery of locomotor function through axonal regeneration following spinal cord injury in rats. J Exerc Rehabil. 2016;12:284-92.

4. Sandrow-Feinberg HR, Houlé JD. Exercise after spinal cord injury as an agent for neuroprotection, regeneration and rehabilitation. Brain Res. 2015;1619:12-21.

5. Young W. Spinal cord regeneration. Cell Transplant. 2014;23: 573-611.

6. Leech KA, Hornby TG. High-intensity locomotor exercise increases brain-derived neurotrophic factor in individuals with incomplete spinal cordInjury. J Neurotraum. 2017;34:1240-8.

7. Ward PJ, Herrity AN, Harkema SJ, Hubscher CH. Traininginduced functional gains following SCI. Neural Plast. 2016;2016: $1-12$.

8. Wang H, Liu N, Zhang YP, Deng L, Lu Q, Shields CB, et al. Treadmill training induced lumbar motoneuron dendritic plasticity and behavior recovery in adult rats after a thoracic contusive spinal cord injury. Exp Neurol. 2015;271:368-78.

9. Hayashibe M, Homma T, Fujimoto K, Oi T, Yagi N, Kashihara $\mathrm{M}$, et al. Locomotor improvement of spinal cord-injured rats through treadmill training by forced plantar placement of hind paws. Spinal Cord. 2015;54:521-9.

10. Smith AC, Knikou M. A review on locomotor training after spinal cord injury: reorganization of spinal neuronal circuits and recovery of motor function. Neural Plast. 2016;2016:1216258.

11. Boyce VS, Mendell LM. Neurotrophic factors in spinal cord injury. Handb Exp Pharmacol. 2014;220:443-60.

12. Harvey AR, Lovett SJ, Majda BT, Yoon JH, Wheeler LP, Hodgetts SI. Neurotrophic factors for spinal cord repair: which, where, how and when to apply, and for what period of time? Brain Res. 2015;1619:36-71.

13. Mitre M, Mariga A, Chao MV. Neurotrophin signalling: novel insights into mechanisms and pathophysiology. Clin Sci. 2017;131:13-23.

14. Weishaupt N, Blesch A, Fouad K. BDNF: the career of a multifaceted neurotrophin in spinal cord injury. Exp Neurol. 2012;238: 254-64.

15. Wu Q, Cao Y, Dong C, Wang H, Wang Q, Tong W, et al. Neuromuscular interaction is required for neurotrophins-mediated locomotor recovery following treadmill training in rat spinal cord injury. PeerJ. 2016;4:e2025.

16. Su YF, Lin CL, Lee KS, Tsai TH, Wu SC, Hwang SL, et al. A modified compression model of spinal cord injury in rats: functional assessment and the expression of nitric oxide synthases. Spinal Cord. 2015;53:432.

17. Waite ME, Tomkovich A, Quinn TL, Schumann AP, Dewberry LS, Totsch SK, et al. Efficacy of common analgesics for postsurgical pain in rats. J Am Assoc Lab Anim Sci. 2015;54:420-5.

18. Kim JY, Choi GS, Cho YW, Cho HK, Hwang SJ, Ahn SH. Attenuation of spinal cord injury-induced astroglial and microglial activation by repetitive transcranial magnetic stimulation in rats. $\mathrm{J}$ Korean Med Sci. 2013;28:295-9.

19. Tashiro S, Shinozaki M, Mukaino M, Renault-Mihara F, Toyama Y, Liu M, et al. BDNF induced by treadmill training contributes to the suppression of spasticity and allodynia after spinal cord injury via upregulation of KCC2. Neurorehab Neural Repair. 2015;29:677-89.

20. Endo T, Ajiki T, Inoue H, Kikuchi M, Yashiro T, Nakama S, et al. Early exercise in spinal cord injured rats induces allodynia through TrkB signaling. Biochem Biophys Res Commun. 2009;381:339-44. 
21. Ying Z, Roy RR, Zhong H, Zdunowski S, Edgerton VR, GomezPinilla F. BDNF-exercise interactions in the recovery of symmetrical stepping after a cervical hemisection in rats. Neuroscience. 2008;155:1070-8.

22. Soril LJJ, Ramer LM, McPhail LT, Kaan TKY, Ramer MS. Spinal brain-derived neurotrophic factor governs neuroplasticity and recovery from cold-hypersensitivity following dorsal rhizotomy. Pain. 2008;138:98-110.

23. Basso DM, Beattie MS, Bresnahan JC. Graded histological and locomotor outcomes after spinal cord contusion using the NYU weight-drop device versus transection. Exp Neurol. 1996;139: 244-56.

24. Basso DM, Beattie MS, Bresnahan JC, Anderson DK, Faden AI, Gruner JA, et al. MASCIS evaluation of open field locomotor scores: effects of experience and teamwork on reliability. Multicenter Animal Spinal Cord Injury Study. J Neurotraum. 1996;13:343-59.

25. Figueroa JD, Cordero K, Baldeosingh K, Torrado AI, Walker RL, Miranda JD, et al. Docosahexaenoic acid pretreatment confers protection and functional improvements after acute spinal cord injury in adult rats. J Neurotrauma. 2012;29:551-66.

26. Cho Y, Ko I, Kim S, Lee S, Shin M, Kim C, et al. Oral mucosa stem cells alleviates spinal cord injury-induced neurogenic bladder symptoms in rats. J Biomed Sci. 2014;21:43.

27. Faul F, Erdfelder E, Lang AG, Buchner A. G*Power 3: a flexible statistical power analysis program for the social, behavioral, and biomedical sciences. Behav Res Methods. 2007;39:175-91.

28. Chuang LL, Chen YL, Chen CC, Li YC, Wong AM, Hsu AL, et al. Effect of EMG-triggered neuromuscular electrical stimulation with bilateral arm training on hemiplegic shoulder pain and arm function after stroke: a randomized controlled trial. J Neuroeng Rehabil. 2017;14:122.

29. Ziemlińska E, Kügler S, Schachner M, Wewiór I, CzarkowskaBauch J, Skup M. Overexpression of BDNF increases excitability of the lumbar spinal network and leads to robust early locomotor recovery in completely spinalized rats. PLoS ONE. 2014;9: e88833.

30. Oh MK, Kim HR, Kim WS, Shin HI. Relationship between motor evoked potential response and the severity of paralysis in spinal cord injury patients. Ann Rehabil Med. 2017;41: 211-7.

31. Namsolleck P, Boato F, Schwengel K, Paulis L, Matho KS, Geurts N, et al. AT2-receptor stimulation enhances axonal plasticity after spinal cord injury by upregulating BDNF expression. Neurobiol Dis. 2013;51:177-91.

32. Duan W, Zhang Y, Hou Z, Huang C, Zhu H, Zhang C, et al. Novel insights into NeuN: from neuronal marker to splicing regulator. Mol Neurobiol. 2016;53:1637-47.

33. Lee AL. Contrasting roles of dynamics in protein allostery: NMR and structural studies of CheY and the third PDZ domain from PSD-95. Biophys Rev. 2015;7:217-26.

34. Kolos YA, Grigoriyev IP, Korzhevskyi DE. A synaptic marker synaptophysin. Morfologiia. 2015;147:78-82.

35. Tartaglia N, Du J, Tyler WJ, Neale E, Pozzo-Miller L, Lu B. Protein synthesis-dependent and -independent regulation of hippocampal synapses by brain-derived neurotrophic factor. J Biol Chem. 2001;276:37585-93.

36. Yoshii A, Constantinepaton M. Postsynaptic localization of PSD95 is regulated by all three pathways downstream of TrkB signaling. Front Synaptic Neurosci. 2014;6:6. 